\title{
Design Guidelines using Selective Harmonic Elimination Advanced Method for DC-AC PWM with the Walsh Transform
}

\author{
Jesus Vicente, Rafael Pindado, Inmaculada Martinez \\ Technical University of Catalonia (UPC) \\ jesus.vicente@upc.edu,rafael.pindado@upc.edu,inmaculada.martinez@upc.edu
}

\begin{abstract}
The use of the Walsh transform in DC-AC PWM waveform generation allows the calculation of the switching angles by means of linear equations which depend on the fundamental amplitude. However, when it is needed a wide regulation of the fundamental amplitude, conventional application of Walsh transform forces to switch among different equation sets, due to the range limitation of each one of them. In this paper it is described an advanced method to obtain the switching angles that permits full regulation of the fundamental amplitude, with only a switching interval vector, in single phase systems. The paper shows Matlab simulation results that proof the efficiency of the advanced method algorithm.
\end{abstract}

Index Terms -Algorithm optimization, PWM inverters, Selective harmonic elimination, DC-AC conversion, Walsh transform.

\section{INTRODUCTION}

In DC-AC PWM waveforms generation with selective harmonic elimination the real time control of the fundamental amplitude takes a great amount of time to compute the nonlinear equations. This question can be solved by the offline calculation of the switching angles, which establishes a compromise between the desired precision and the memory storage required.

On the other hand, it is possible the on-line calculation of the switching angles as a linear function of the fundamental amplitude using the Walsh functions [1]. These functions permit the linearization of the equation set that leads to the harmonic cancellation [2-4].

The Walsh functions, defined by J.L.Walsh in 1923, are made of a set of orthogonal and orthonormal functions that only have two values, $\{+1-1\}$. In this paper the functions are normalized in time (unity period). As an example, Fig. 1 depicts the 17 first Walsh functions subset.

Every periodic function $\mathrm{f}(\mathrm{t})$ may be expressed as a sum of Walsh functions as:

$$
f(t)=W_{0} \operatorname{wal}(0, t)+\sum_{k=1}^{\infty} W_{k} \operatorname{wal}(k, t), t \in[0,1)
$$

where:

$$
\begin{gathered}
W_{0}=\int_{0}^{1} f(t) \operatorname{wal}(0, t) d t=\int_{0}^{1} f(t) d t \\
W_{k}=\int_{0}^{1} f(t) \text { wal }(k, t) d t
\end{gathered}
$$

For evident reasons Walsh series expansion is especially useful for the representation of PWM waveforms. In particular, as PWM waveforms generated in DC-AC converters have half-wave symmetry its series expansion will only have Walsh functions with $4 n-3$ order, where $n$ is an integer $\{n=1,2,3,4, \ldots\}$.

$$
f(t)=\sum_{n=1}^{\infty} W_{(4 n-3)} \operatorname{wal}(4 n-3, t)
$$

With this technique the use of $\mathrm{M}$ angles per quarter of period permits the cancellation of M-1 harmonics and the regulation of the fundamental amplitude.

However, this technique has the drawback of obtaining a great number of solutions that difficult the selection process of the better cases and also increments the computation time, especially when a big number of switching angles is used.

On the other hand, there are different solutions for each value of fundamental amplitude depending on the position of the switching angles in the intervals of the first quarter period (switching interval vector). Obviously, these solutions cause different harmonic distributions.

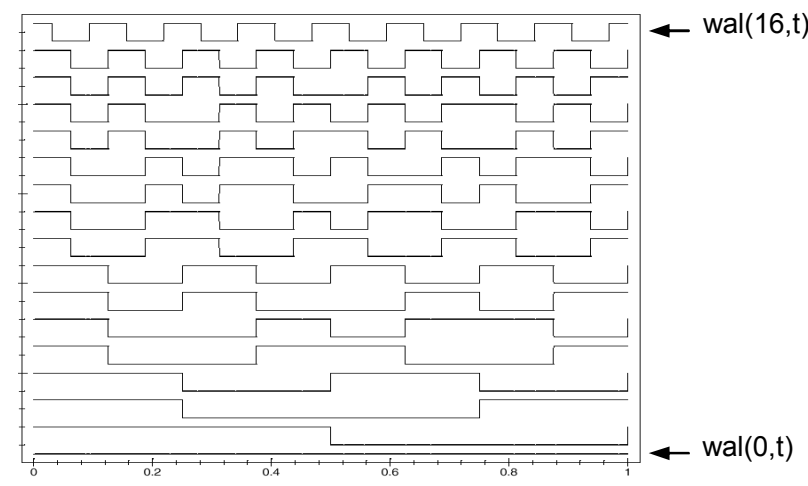

Fig.1. Walsh functions: wal(i,t),$i \in\{0, . ., 16\}$

In order to compare the differences in these harmonic distributions, the distortion factor defined in (3) has been chosen as the parameter to be considered. This factor is preferred to the THD, because the harmonic order, k, diminishes the influence of the harmonic amplitude. This behavior is analogous to the filtering obtained when an inductive load is connected to the inverter. In this paper, the maximum order selected to do the computing is $\mathrm{k}=39$. 


$$
D F=\frac{100}{A_{1}}\left(\sum_{k=3}^{k \rightarrow \infty}\left(\frac{A_{k}}{k}\right)^{2}\right)^{1 / 2}
$$

The efficiency of the control algorithm of the inverter will be better if it achieves a wide fundamental range, a low distortion factor and a small processing time.

In the following sections there is a comparison between the conventional Walsh transform method and the advanced method, proposed by the authors [5].

\section{CONVENTIONAL METHOD ANALYSIS}

Since the problem of harmonic elimination is inherent to the frequency domain, the analysis requires the availability of reciprocal conversion tools between Walsh and Fourier transforms. The relationship between Fourier and Walsh coefficients is the starting point to compute the switching angles of the PWM waveform and can be expressed by the transformation (4) developed in references [2], [3], [6], where $\boldsymbol{G}_{\boldsymbol{F}}$ and $\boldsymbol{G}_{\boldsymbol{W}}$ are the coefficients of the PWM signal by Fourier and Walsh expansions respectively, and $\boldsymbol{B}$ is the FourierWalsh conversion matrix .

$$
G_{F}=B G_{W}
$$

The Walsh coefficients are obtained as follows:

$$
G_{W}=C \phi+D
$$

where $\phi=\left[\begin{array}{llll}\phi_{1} & \phi_{2} & \ldots & \phi_{M}\end{array}\right]^{T}$ is the vector of switching angle fractions, each one referred to the end of its switching interval, $\phi_{i} \in(0,1)$, shown in figure 2 , while $\boldsymbol{C}$ and $\boldsymbol{D}$ are the set of coefficients that relate the angles with the Walsh expansion coefficients.

Each quarter period is subdivided in $\mathrm{N}$ intervals, from 0 to $\mathrm{N}-1$, but only $\mathrm{M}$ of which include one, and only one, switching angle. Those intervals $m\left(\alpha_{i}\right)$ form the elements of the switching interval vector (6).

$$
\boldsymbol{m}=\left[m\left(\alpha_{1}\right) m\left(\alpha_{2}\right) \ldots m\left(\alpha_{M}\right)\right]
$$

To simplify the notation the elements of vector $\mathbf{m}$ would be referred as follows:

$$
\boldsymbol{m}=[m(1) m(2) \ldots m(M)]
$$

The relationship between the fractions $\left(\phi_{i}\right)$ and the switching angles $\left(\alpha_{\mathrm{i}}\right)$ is given by (8) and can be observed in figure 2 .

$$
\alpha_{\mathrm{i}}=\frac{\pi}{2 N}\left(m(i)+1-\phi_{\mathrm{i}}\right)
$$

Fixing $\mathrm{N}$ as the power of 2 greater or equal to 4 times the number of angles, it can be made that all Walsh functions used in the expansion of the PWM signal have a constant value in each subinterval, reducing the complexity of the algorithm. Another simplification can be obtained by fixing the end of the switching $\left(\beta_{\mathrm{i}}\right)$ in the next interval if the value of $\mathrm{m}(\mathrm{i})$ is less than $(\mathrm{N} / 2)-1$, and in the same interval otherwise [2].

Fig. 2 shows the first quarter of a PWM signal with two switching angles $\left(\alpha_{1}, \alpha_{2}\right)$ at intervals 2 and 6 . In this case: $\mathrm{M}=2, \mathrm{~N}=8, \mathrm{~m}(1)=2$ and $\mathrm{m}(2)=6$.

Taking this into account, matrix $\mathbf{C}$ and column vector $\mathbf{D}$ are easily derived from the Walsh matrix (WAL) whose elements are obtained from (9).

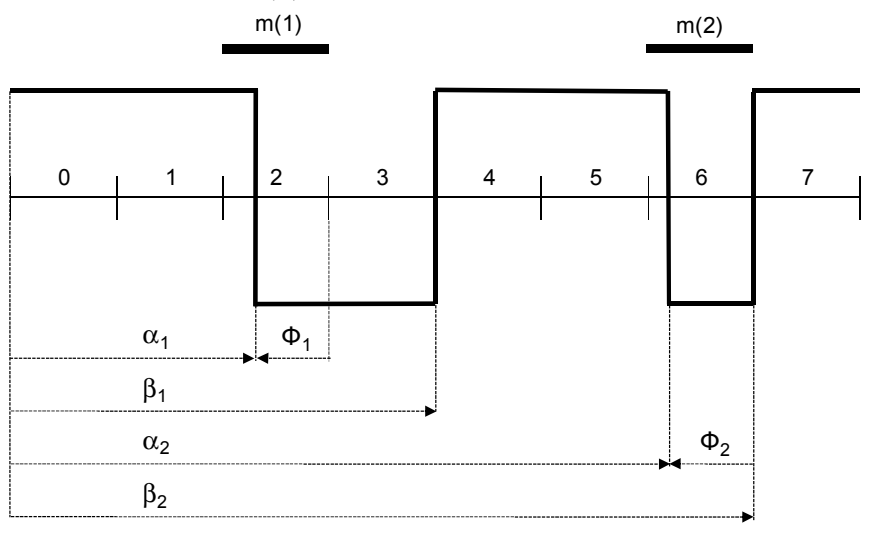

Fig. 2. PWM signal with two switching angles (conventional method).

$$
\mathrm{WAL}_{\mathrm{i}, \mathrm{j}}=\operatorname{wal}\left(4 i-3, \frac{j-1}{4 N}\right) \quad i, j=1 \ldots N
$$

Matrix $\boldsymbol{C}$ is formed with the $\mathrm{M}$ columns of WAL corresponding to vector $\mathbf{m}$, multiplied by the factor $2 / \mathrm{N}$.

$$
\mathrm{C}_{\mathrm{i}, \mathrm{j}}=-\frac{2}{N} \mathrm{WAL}_{\mathrm{i}, \mathrm{m}(\mathrm{j})+1} \quad i=1 \ldots N, j=1 \ldots M
$$

The N-dimensional column vector $\boldsymbol{D}$ is obtained by (11).

$$
\mathrm{D}_{\mathrm{i}}=\frac{1}{\mathrm{~N}}\left[\sum_{\mathrm{j}=1}^{\mathrm{N}} \mathrm{WAL}_{\mathrm{i}, \mathrm{j}}-2 \sum_{\mathrm{j}=1}^{\mathrm{M}} \mathrm{PW}_{\mathrm{i}, \mathrm{m}(\mathrm{j})+1}\right] \quad i=1 \ldots N
$$

Matrix PW is obtained by shifting left one column matrix WAL and making zero the columns whose index is greater than $(\mathrm{N} / 2)-1$.

Combining equations (4) and (5) we obtain (12):

$$
G_{F}=B(C \phi+D)=E \phi+F
$$

We can obtain the linear system for the switching angles from (13):

$$
\phi=E^{-1}\left(G_{F}-F\right)
$$

Now, leaving the fundamental amplitude $\left(A_{1}\right)$ variable, and making zero the rest of the harmonics $\left(\mathrm{A}_{3}, \mathrm{~A}_{5}, \ldots\right)$, the linear system is represented by (14): 


$$
\phi=\boldsymbol{P} A_{1}+K
$$

This linear system has the restrictions that $\mathrm{A}_{1}$ and $\phi_{i} \in[0,1]$. Also, in order to validate the equations, the range of fundamental amplitude should be positive.

\section{ADVANCED METHOD ANALYSIS}

With the advanced method, the PWM signal has notches placed in adjacent intervals and symmetry properties that are clearly shown in Fig. 3. In this case the end of the switching $\left(\beta_{i}\right)$ is variable.

As it will be shown this way of generating the PWM signal makes it possible to achieve ranges of variation of the fundamental amplitude that are near to $100 \%$, value that is impossible to reach with the conventional method.

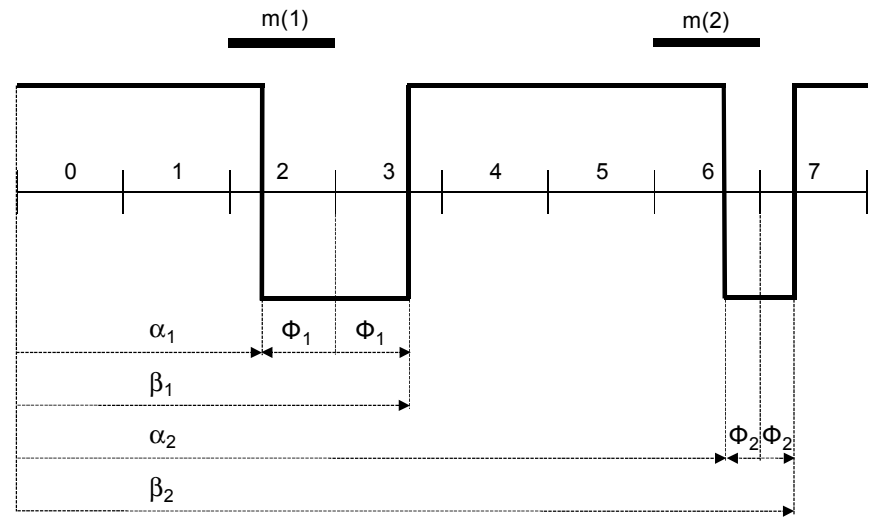

Fig. 3. PWM signal with two switching angles (advanced method).

Again, the Walsh coefficients are obtained from (15) as it has been shown in (5) but the matrices $\mathbf{C}$ and $\mathbf{D}$ will differ.

$$
G_{W}=C \phi+D
$$

where $\phi=\left[\begin{array}{llll}\phi_{1} & \phi_{2} & \ldots & \phi_{M}\end{array}\right]^{T}$ is the vector of switching angle fractions, referred to the center of the notches. $\phi_{i} \in(0,1)$. The relationship between these fractions and the switching angles $\left(\alpha_{i}\right)$ is given by (16).

$$
\alpha_{i}=\frac{\pi}{2 N}\left(m(i)+1-\phi_{i}\right)
$$

The end of the switching $\left(\beta_{\mathrm{i}}\right)$ is given by (17).

$$
\beta_{i}=\frac{\pi}{2 N}\left(m(i)+1+\phi_{i}\right)
$$

These changes produce a notable simplification of the expressions needed to find the switching vectors which have solution.

$$
\begin{aligned}
& C_{i, j}=-\frac{2}{N}\left(W A L_{i, m(j)}+W A L_{i, m(j)+1}\right) \\
& i=1 \ldots N, j=1 \ldots M
\end{aligned}
$$

$$
D_{i}=\frac{1}{N} \sum_{j=1}^{N} W A L_{i, j}
$$

Notice the simplification achieved in the computation of the elements of matrix $\mathbf{D}$.

Following the same reasoning done with the conventional method the linear system that links the angle fractions with the fundamental amplitude will be:

$$
\phi=P A_{1}+K
$$

\section{RESULTS COMPARISON}

In order to compare the methods described above, an exhaustive process has been developed. Several questions have to be considered.

First of all, figure 4 shows the number of interval combinations tested and the computing time needed to find which of them have a positive range of variation of the fundamental amplitude, as a function of the angles number. It can be seen that the processing times are similar in both methods. The processor used was an Intel T5270 CPU working at a frequency of $1.4 \mathrm{GHz}$.

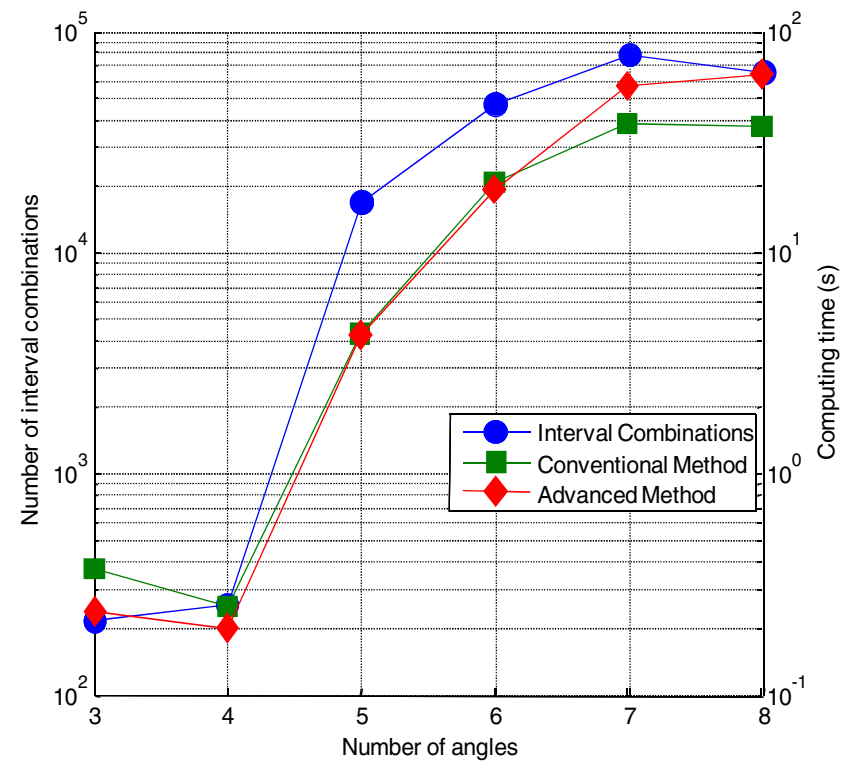

Fig. 4. Number of interval combinations and computing time.

Secondly, figure 5 shows the results obtained using the conventional and the advanced Walsh transform methods, respectively. This figure shows the solutions for each number of angles. The columns grouped to the left are for the conventional method $(\mathrm{C})$ and the columns to the right are for the advanced method (A). It can be observed that the advanced method achieves greater ranges of variation of the fundamental amplitude. 


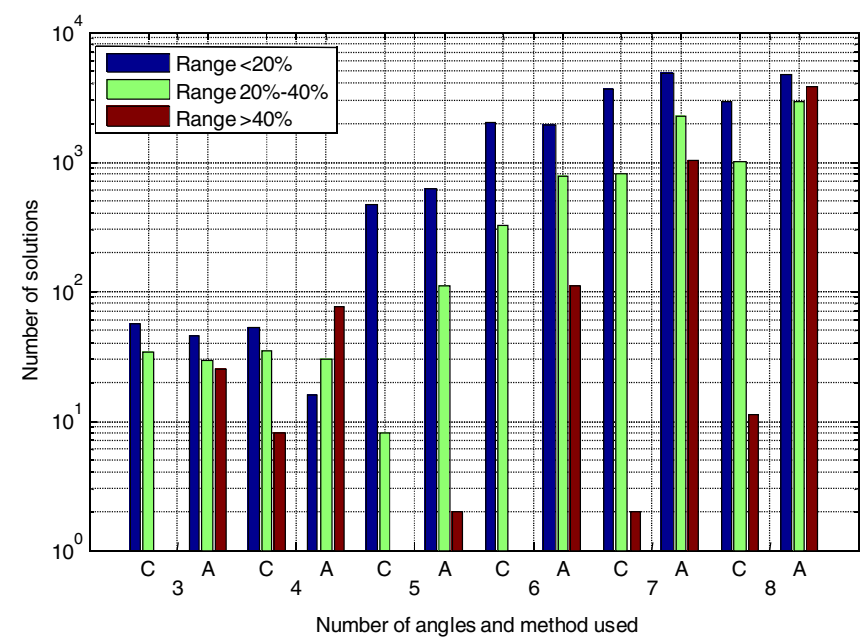

Fig. 5. Number of solutions obtained.

In tables I and II it can be seen the minimum and maximum values of fundamental amplitude (per unit), considering all the solutions. They also show the maximum fundamental range available with a single switching interval. Finally the values of distortion factor are shown, making the calculation when the fundamental amplitude is the maximum in the switching interval.

TABLE I

Amplitude Range and Distortion Factor for Conventional Method

\begin{tabular}{|c|c|c|c|cc|}
\hline $\begin{array}{c}\text { Number of } \\
\text { angles }\end{array}$ & A1 & A1 & A1 & \multicolumn{2}{|c|}{ DF (\%) } \\
\hline 8 & 0.53 & 1.01 & 43.9 & 1.8 & 6.6 \\
\hline 7 & 0.56 & 1.02 & 40.1 & 2.3 & 7.5 \\
\hline 6 & 0.65 & 1.02 & 32.9 & 2.5 & 6.5 \\
\hline 5 & 0.77 & 1.03 & 24.2 & 2.9 & 7.3 \\
\hline 4 & 0.46 & 1.04 & 51.0 & 3.8 & 16.2 \\
\hline 3 & 0.60 & 1.06 & 43.4 & 4.7 & 15.6 \\
\hline
\end{tabular}

TABLE II

Amplitude Range and Distortion Factor for Advanced Method

\begin{tabular}{|c|c|c|c|cc|}
\hline $\begin{array}{c}\text { Number of } \\
\text { angles }\end{array}$ & $\begin{array}{c}\mathrm{A} 1 \\
\min \end{array}$ & $\mathrm{A} 1$ & $\mathrm{~A} 1$ & \multicolumn{2}{|c|}{$\mathrm{DF}(\%)$} \\
\hline 8 & 0.06 & 1.01 & 94.3 & 1.5 & 3.8 \\
\hline 7 & 0.23 & 1.02 & 78.7 & 1.8 & 5.3 \\
\hline 6 & 0.39 & 1.02 & 62.4 & 2.3 & 8.1 \\
\hline 5 & 0.57 & 1.03 & 45.0 & 2.8 & 8.1 \\
\hline 4 & 0.03 & 1.04 & 98.6 & 3.7 & 18.7 \\
\hline 3 & 0.32 & 1.07 & 74.2 & 4.8 & 19.8 \\
\hline
\end{tabular}

It should be remarked that the values shown are not for the same switching interval vector. This is the reason why, for 8 angles in the conventional method, although a fundamental minimum value of 0.53 and a maximum of 1.01 can be obtained, the maximum fundamental range is, only, $43.9 \%$.

It can be seen that the advanced method achieves a wider range of amplitudes, with similar values of distortion factor. The best results in amplitude range, for the advanced method, are obtained for 8 and 4 angles, being the corresponding switching interval vectors: $\mathbf{m}=\left[\begin{array}{llllllll}2 & 6 & 10 & 14 & 18 & 22 & 26 & 30\end{array}\right]$ and $\mathbf{m}=\left[\begin{array}{llll}1 & 5 & 9 & 13\end{array}\right]$. In both cases the number of angles is a power of two and the intervals are equally distributed in the first quarter of the period.

\section{SiMULATION EXAMPLES}

In this section some simulation examples for 4 and 8 angles are described, for the two methods discussed above. The switching intervals chosen are those which have the greatest range of variation for the fundamental amplitude.

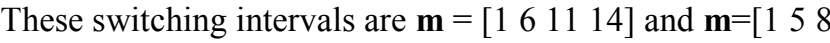
$\left.\begin{array}{lllll}13 & 19 & 21 & 26 & 29\end{array}\right]$ with the conventional method and $\mathbf{m}=\left[\begin{array}{lll}1 & 5 & 9\end{array}\right.$ 13] and $\mathbf{m}=\left[\begin{array}{llllllll}2 & 6 & 10 & 14 & 18 & 22 & 26 & 30\end{array}\right]$ using the advanced method. For this switching intervals, in tables III to VI there are represented the linear equations for the angles (21)-(24) and the minimum and maximum values that can be obtained for $A_{1}$.

TABLE III

Equations And Amplitude RANGe Conventional Method

\begin{tabular}{|c|}
\hline $\mathrm{m}=[161114]$ \\
\hline$\Phi_{1}=-0.3590 \mathrm{~A}_{1}+1.1490$ \\
$\Phi_{2}=-1.5803 \mathrm{~A}_{1}+1.5483$ \\
$\Phi_{3}=-1.8984 \mathrm{~A}_{1}+1.8922$ \\
$\Phi_{4}=-1.3055 \mathrm{~A}_{1}+1.3941$ \\
\hline $\mathrm{A}_{1} \min =47.00 \%, \mathrm{~A}_{1} \max =97.97 \%$ \\
\hline
\end{tabular}

TABLE IV

EQuATIONS AND AmPLitude RANGe AdVANCEd Method $\mathrm{m}=\left[\begin{array}{llll}1 & 5 & 9 & 13\end{array}\right]$

$\Phi_{1}=-0.1954 \mathrm{~A}_{1}+0.9018$

$\Phi_{2}=-0.5565 \mathrm{~A}_{1}+1.0187$

$\Phi_{3}=-0.8328 \mathrm{~A}_{1}+0.9938$

$\Phi_{4}=-0.9824 \mathrm{~A}_{1}+1.0016$

$\mathrm{A}_{1} \min =3.37 \%, \mathrm{~A}_{1} \max =101.96 \%$

TABLE $\mathrm{V}$

EQUATIONS AND AmPLITUde RANGe CONVENTIONAL METHOD

\begin{tabular}{|c|}
\hline $\mathrm{m}=[1581319212629]$ \\
\hline$\Phi_{1}=0.7935 \mathrm{~A}_{1}+0.1434$ \\
$\Phi_{2}=-1.2594 \mathrm{~A}_{1}+1.4115$ \\
$\Phi_{3}=-0.1541 \mathrm{~A}_{1}+0.2644$ \\
$\Phi_{4}=-2.1312 \mathrm{~A}_{1}+2.1001$ \\
$\Phi_{5}=-1.3492 \mathrm{~A}_{1}+1.6192$ \\
$\Phi_{2}=-1.7046 \mathrm{~A}_{1}+1.9318$ \\
$\Phi_{7}=-1.7874 \mathrm{~A}_{1}+1.8833$ \\
$\Phi_{8}=-2.0562 \mathrm{~A}_{1}+2.0576$ \\
\hline $\mathrm{A}_{1} \min =54.66 \%, \mathrm{~A}_{1} \max =98.4 \%$ \\
\hline
\end{tabular}

TABLE VI

EQUATIONS AND AMPLITUDE RANGE ADVANCED METHOD

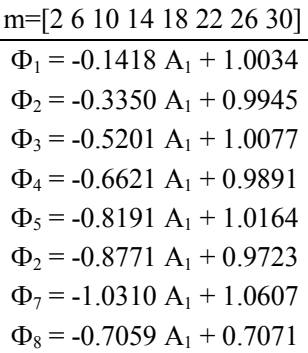

$\mathrm{A}_{1} \min =5.88 \%, \mathrm{~A}_{1} \max =100.18 \%$ 

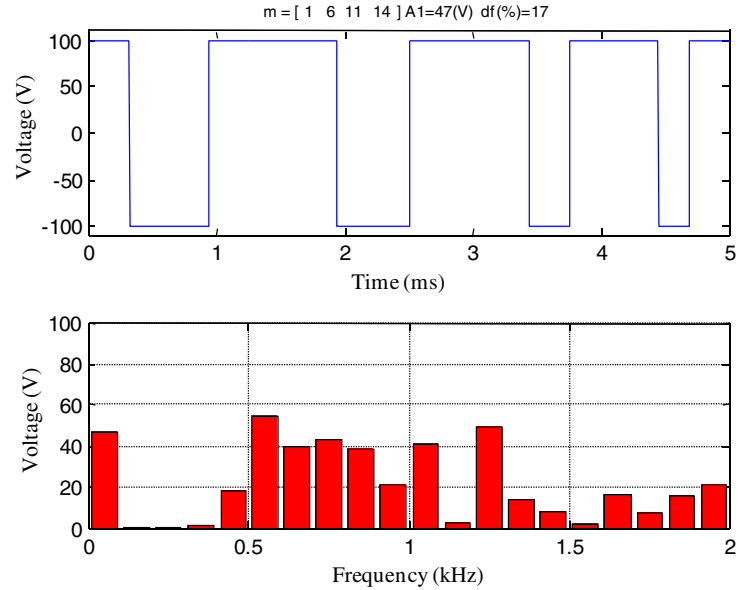

(a)
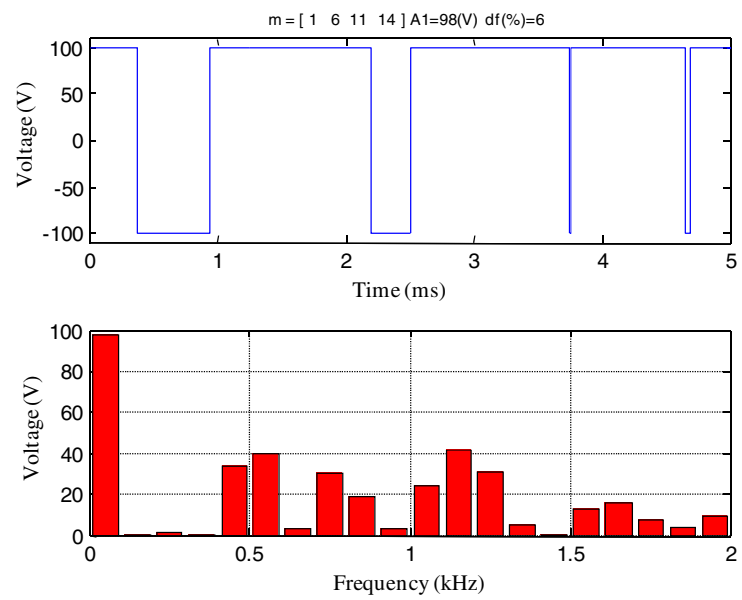

Fig. 6. 4 angles, PWM signal and harmonic distribution. Conventional Method. (a) $\mathrm{A}_{1}=47 \%$, (b) $\mathrm{A}_{1}=98 \%$

Simulation results for 4 angles are shown in figures 6 (Conventional Method) and 7 (Advanced Method).

In order to compare more faithfully the harmonic distributions and the distortion factors, there have been taken the values $47.00 \%$ and $97.97 \%$ that are the minimum and maximum A1 values for the conventional method.

The obtained values of distortion factors using the advanced method are $15 \%$ and $5 \%$, for the lower and higher fundamental amplitudes, respectively. Using the conventional method the obtained values are $17 \%$ and $6 \%$, respectively.

Figures 8 (Conventional Method) and 9 (Advanced Method) show the simulation results for 8 angles. As before, there have been taken the fundamental amplitude values of $54.66 \%$ and $98.40 \%$, to compare the distortion factor and the harmonic distribution.

The obtained values of distortion factors using the advanced method are $6 \%$ and $2 \%$, for the lower and higher fundamental amplitudes, respectively. Using the conventional method the obtained values are $7 \%$ and $3 \%$, respectively.
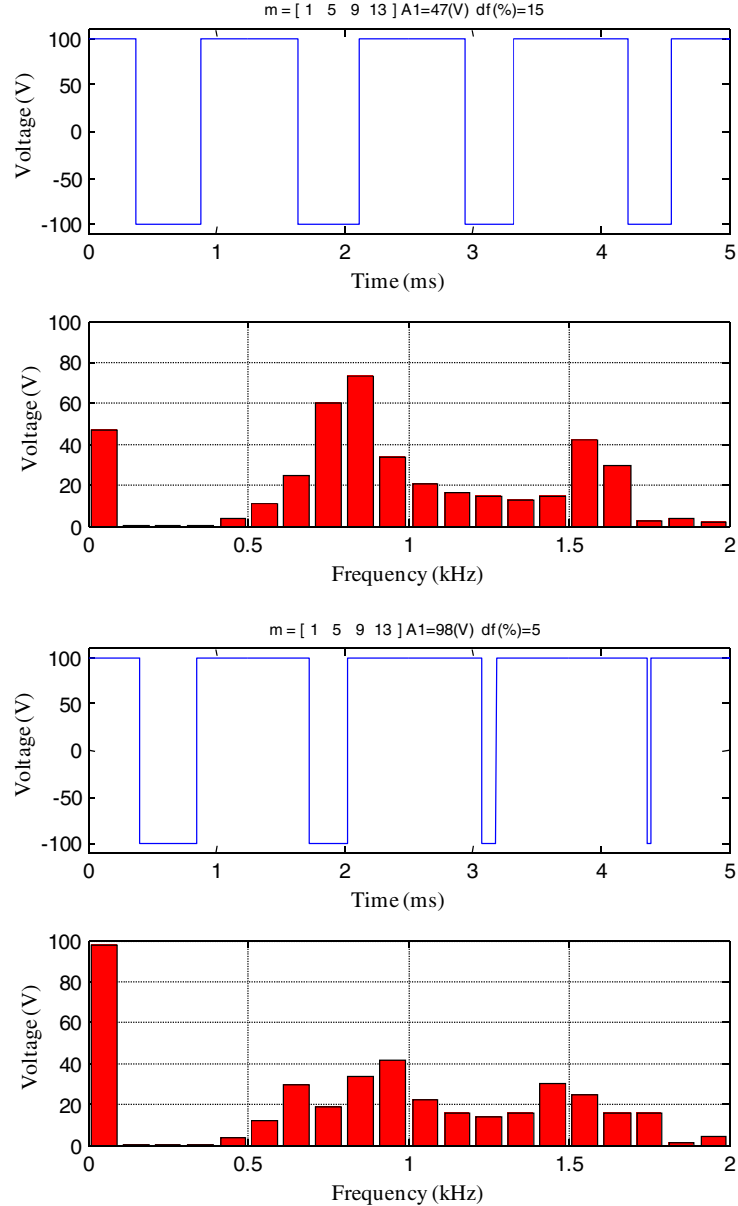

(b)

Fig. 7. 4 angles, PWM signal and harmonic distribution. Advanced Method. (a) $\mathrm{A}_{1}=47 \%$, (b) $\mathrm{A}_{1}=98 \%$

It can be seen that the PWM waveforms obtained from the advanced method have a regular shape: the notches are thinner as they approach the quarter of period. Also the distortion factors for the same $\mathrm{A}_{1}$ values are smaller when the advanced method solution is taken.

Also, figure 9, shows that the orders of the two harmonics with greatest peak amplitudes are 31 and 33, that complies with equation (25). This fact is also verified using advanced method, with 4, 16, 32 and 64 angles when the switching interval vectors are $[2: 4: 4 \cdot \mathrm{M}]$.

$$
k_{\text {peak }}=4 \cdot M \pm 1
$$

\section{DESIGN GUIDELINES}

The following paragraphs give the design guidelines.

First, select the distortion factor tolerable in the particular application in agreement with the required fundamental amplitude. This leads to choose a number of angles that cancels enough harmonics as seen in tables I and II. 

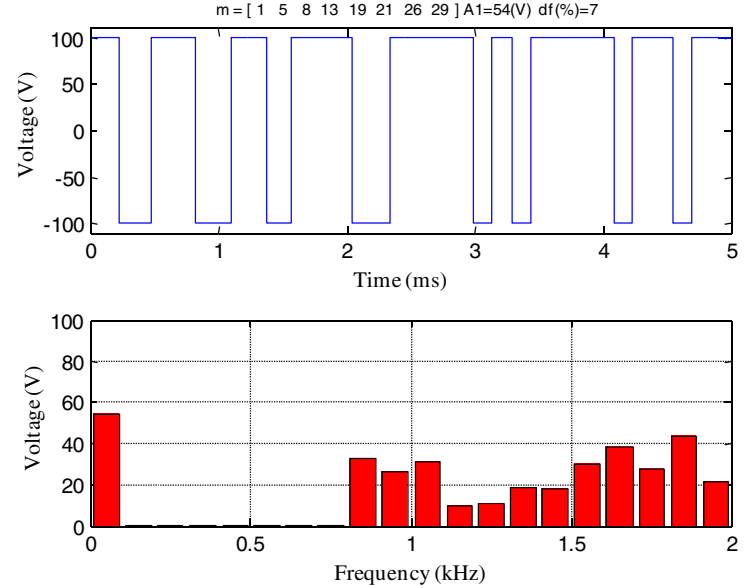

(a)
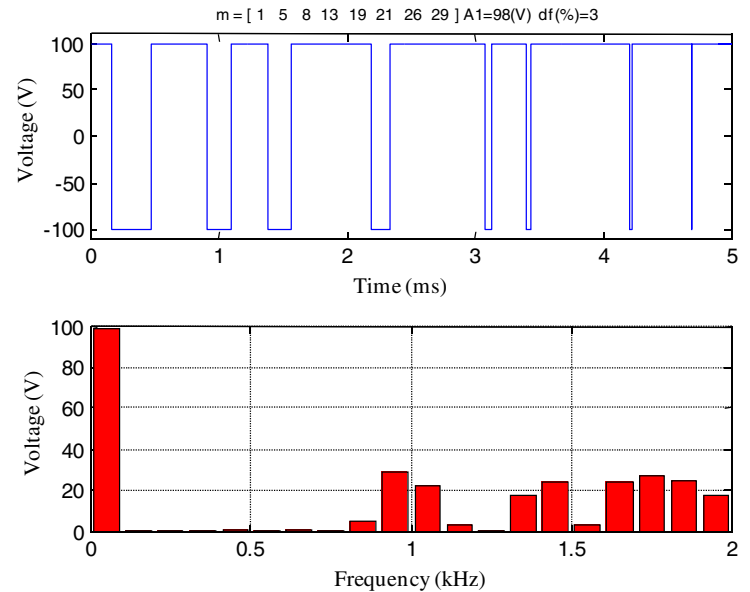

(b)

Fig. 8.8 angles. PWM signal and harmonic distribution. Conventional Method. (a) $\mathrm{A}_{1}=54 \%$, (b) $\mathrm{A}_{1}=98 \%$

Another consideration is the range of fundamental amplitude needed. When a wide range is necessary, it should be chosen the advanced method.

Finally, when a big amount of harmonics should be cancelled, the advanced method gives the best solutions for the switching interval vectors whose number of angles is a power of two and the intervals are equally distributed in the first quarter of the period.

\section{CONCLUSION}

An advanced algorithm has been presented, based on the Walsh functions. This algorithm produces a set of linear equations that allows a wide range of fundamental amplitude regulation, near 100 per cent, with a single switching vector, reducing the processing time needed for the computation of the switching angles for the required output voltage.

Another significant contribution of this work is the design considerations to guide in the selection of the switching vector value for the optimal performance of the converter, obtaining the maximum regulation range of the fundamental component.
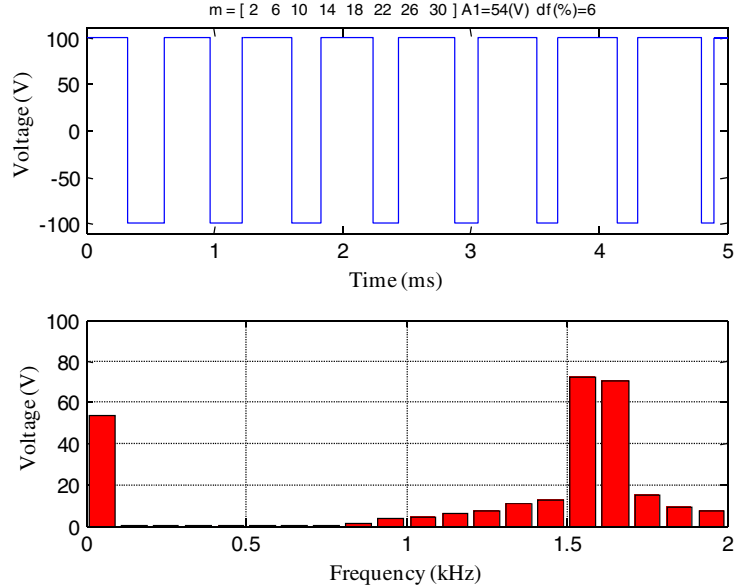

(a)
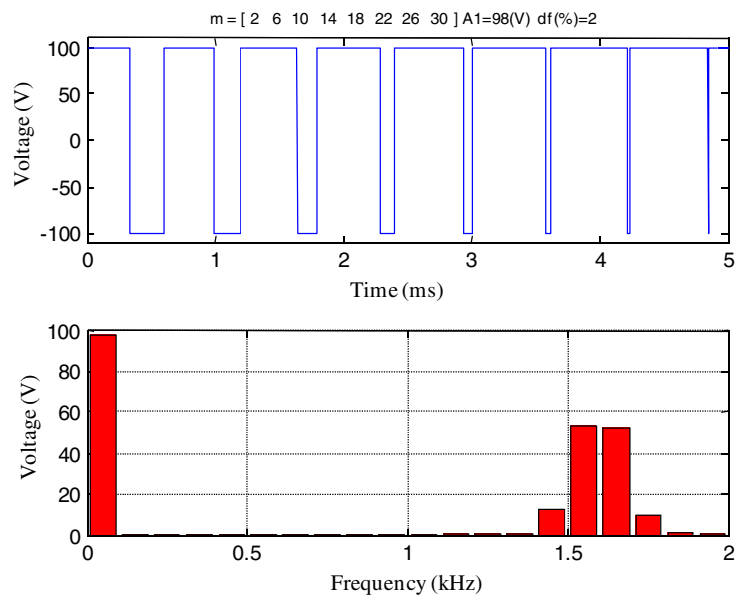

(b)

Fig. 9.8 angles. PWM signal and harmonic distribution. Advanced Method. (a) $\mathrm{A}_{1}=54 \%$, (b) $\mathrm{A}_{1}=98 \%$

\section{REFERENCES}

[1] T.J. Rivlin and E.B. Saff, Joseph L. Walsh: selected papers, New York: Springer, 2000.

[2] F. Swift and A. Kamberis, "A New Walsh Domain Technique of Harmonic Elimination and Voltage Control in Pulse Width Modulated Inverters". IEEE Trans. Power Electron., vol. 8, no. 2, pp.170-185, April 1993.

[3] T.J. Liang, R.M. O'Connell and R.G Hoft, "Inverter Harmonic Reduction Using Walsh Function Harmonic Elimination Method", IEEE Trans. Power Electron., vol. 12, no. 6, pp. 971-982, Nov. 1997.

[4] J. Vicente, R. Pindado and I. Martinez. "Algorithm optimization for PWM signal generation with selective harmonic elimination using the Walsh transform". International Conference on Renewable Energy and Power Quality, proceedings. ICREPQ'03. ISBN 84-607-7173-3, 9-12 April 2003.

[5] J. Vicente, R. Pindado, I. Martinez and J. Pou, "A new efficient algorithm for DC-AC PWM waveform generation with full fundamental regulation on a single linear equation set," Industrial Electronics Society, 2003. IECON '03. The 29th Annual Conference of the IEEE, vol.2, no., pp. 1835-1839 Vol.2, 2-6 Nov. 2003.

[6] K.H. Siemens and R. Kitai, "A Nonrecursive Equation for the Fourier Transform of Walsh Function", IEEE Trans. Electromagn. Compat., vol. 15, no. 2, pp. 81-83, May 1973. 